E. M. ShOKRY (Cairo)

A. N. Ahmed (Cairo)

E. A. Rakha (Suez)

H. M. Hewedi (Suez)

\title{
NEW RESULTS ON THE NBUFR AND NBUE CLASSES OF LIFE DISTRIBUTIONS
}

Abstract. Some properties of the "new better than used in failure rate" (NBUFR) and the "new better than used in expectation" (NBUE) classes of life distributions are given. These properties include moment inequalities and moment generating functions behaviors. In addition, nonparametric estimation and testing of the survival functions of these classes are discussed.

1. Introduction. Let $X \geq 0$ be a random life with distribution function (df) $F$ and survival function (sf) $\bar{F}=1-F$. Assume that $X$ has a finite mean $\mu=E(X)=\int_{0}^{\infty} \bar{F}(x) d x$. Thus throughout this paper it is assumed that $0<\mu<\infty$.

In practice, $X$ is often assumed to be (but need not be) absolutely continuous with probability density function (pdf) $f(x)$. One of the commonly applied concepts of positive aging which is based on a property of the failure rate $r(t)$ of the distribution is the NBUFR class of life distributions. This class is defined and studied in [5] and [1].

Precisely, we have the following definition:

Definition 1.1 ([1]). An absolutely continuous distribution function $F$ for which $\lim _{x \rightarrow 0+} F(x) / x$ exists is said to be new better (resp. worse) than used in failure rate, denoted by NBUFR (resp. NWUFR), if

$$
\bar{F}(x+t) \leq(\geq) \bar{F}(x) e^{-r(0) t} \quad \text { for all } x, t \geq 0,
$$

2000 Mathematics Subject Classification: Primary 62N05; Secondary 60E15, 62G05, $62 \mathrm{G} 10$.

Key words and phrases: new better than used in failure rate, moment inequalities, moment generating function, estimation and life testing, Pitman asymptotic efficacy. 
where $r(t)=f(t) / \bar{F}(t)$ is the failure rate of $F$ and $f$ is the density corresponding to $F$. Equation (1.1) is equivalent to $r(t) \geq(\leq) r(0)$ for all $t \geq 0$.

The NBUFR class is wider than NBU, since every NBU (resp. NWU) distribution is NBUFR (resp. NWUFR). It is known that any $k$-out-of- $n$, $1 \leq k<n$, system has the NBUFR property (see [1]), a property that only the NBUFR enjoys among all known aging classes.

The present work addresses additional probability and inferential properties of the NBUFR and NWUFR classes. These properties have not been dealt with before. In Section 2, we give moment inequalities for these two classes. Section 3 gives upper bounds for the moment generating functions of the two classes that guarantee their existence and finiteness. In Section 4, we estimate $\bar{F}(x)$ whenever assumed to be NWUFR and demonstrate its consistency with the rate of convergence. Finally, testing in these classes is discussed in Section 5 .

2. Moment inequalities. The following result gives the moment inequality for the NBUFR class.

TheOREM 2.1. Let $F$ be NBUFR such that for some integers $r, s \geq 0$, $\mu_{(r+s+2)}=E\left(X^{r+s+2}\right)<\infty$. If $\lambda_{(r)}=\mu_{(r)} / r$ !, $r \geq 0$, then

$$
\lambda_{(r+s+2)} \leq \lambda_{(r+1)}[1 / r(0)]^{s+1} .
$$

Proof. Since F is NBUFR, we have

$$
\bar{F}(x+t) \leq \bar{F}(x) e^{-r(0) t} .
$$

Thus for all integers $r, s \geq 0$,

$$
\int_{0}^{\infty} \int_{0}^{\infty} x^{r} t^{s} \bar{F}(x+t) d x d t \leq \int_{0}^{\infty} x^{r} \bar{F}(x) d x \int_{0}^{\infty} t^{s} e^{-r(0) t} d t .
$$

The right-hand side of $(2.1)$ is equal to

$$
\begin{aligned}
{[1 / r(0)]^{s+1} s ! \int_{0}^{\infty} x^{r} \bar{F}(x) d x } & =[1 / r(0)]^{s+1} s ! E \int_{0}^{X} x^{r} d x \\
& =\frac{\mu_{(r+1)}}{r+1} s ![1 / r(0)]^{s+1}
\end{aligned}
$$

The left-hand side of (2.1) is equal to

$$
\begin{aligned}
& \int_{0}^{\infty} \int_{0}^{u}(u-v)^{r} v^{s} \bar{F}(u) d v d u=\int_{0}^{\infty} u^{r+s+1} \bar{F}(u) d u \int_{0}^{1} w^{s}(1-w)^{r} d w \\
& \quad=\beta(r+1, s+1) \int_{0}^{\infty} u^{r+s+1} \bar{F}(u) d u=\frac{\mu_{(r+s+2)}}{(r+s+2)} \frac{r ! s !}{(r+s+1) !} .
\end{aligned}
$$

The result follows from (2.2) and (2.3). 
REMARK 2.1. Theorem 2.1 above may be extended as follows. The definition of NBUFR is equivalent to the following. Let $x_{1}, \ldots, x_{k+1}$ be nonnegative. Then $F$ is NBUFR if and only if

$$
\bar{F}\left(\sum_{i=1}^{k+1} x_{i}\right) \leq \bar{F}\left(x_{1}\right) e^{-r(0) \sum_{i=2}^{k+1} x_{i}} .
$$

Using the same methodology, if $F$ is NBUFR then

$$
\lambda_{\left(\sum_{i=1}^{k+1} r_{i}+k+1\right)} \leq \lambda_{(r+1)}[1 / r(0)]^{\sum_{i=2}^{k+1} r_{i}+k} .
$$

We state the following interesting special cases:

Corollary 2.1. If $r=s=0$, then $\mu_{(2)} \leq 2 \mu_{(1)} / r(0)$.

Corollary 2.2. If $s=0$, then $\mu_{(r+2)} \leq(r+2) \mu_{(r+1)} / r(0)$.

Corollary 2.3. If $r=0$, then $\mu_{(s+2)} \leq(s+2) ! \mu_{(1)}[1 / r(0)]^{s+1}$.

Next, we give an analogous result for the NBUE class. Toward this end we define the equilibrium life time $\tilde{X}$ whose sf is defined by

$$
\bar{F}_{(1)}(x)=\frac{1}{\mu} \int_{x}^{\infty} \bar{F}_{X}(u) d u .
$$

It can be shown that $F$ is NBUFR if and only if $F_{(1)}$ is NBUE. Using this result, we can prove the following theorem.

TheOREM 2.2. Let $F$ be NBUE such that for some integers $r, s \geq 0$, $\mu_{(r+s+3)}=E\left(X^{r+s+3}\right)<\infty$. If $\lambda_{(r+1)}=\mu_{(r+1)} /(r+1) !, r \geq 0$, then $\lambda_{(r+s+3)} \leq \lambda_{(r+2)}[\mu]^{s+1}$.

Proof. Let $\tilde{\mu}_{(r)}$ denote the $r$ th moment of $\tilde{X}$. Then

$$
\begin{aligned}
\tilde{\mu}_{(r)} & =r \int_{0}^{\infty} x^{r-1} \bar{F}_{(1)}(x) d x=\frac{r}{\mu} \int_{0}^{\infty} x^{r-1} \int_{x}^{\infty} \bar{F}_{X}(u) d u d x \\
& =\frac{r}{\mu} \int_{0}^{\infty} \bar{F}_{X}(u) \int_{0}^{u} x^{r-1} d x d u=\frac{1}{\mu} \int_{0}^{\infty} u^{r} \bar{F}_{X}(u) d u \\
& =\frac{1}{\mu} E \int_{0}^{X} x^{r} d x=\frac{\mu_{(r+1)}}{(r+1) \mu} .
\end{aligned}
$$

But $F$ is NBUFR, so $F_{(1)}$ is NBUE and thus using Theorem 2.1 we get

$$
\frac{\tilde{\mu}_{(r+s+2)}}{(r+s+2) !} \leq \frac{\tilde{\mu}_{(r+1)}}{(r+1) !}[\mu]^{s+1},
$$

since $r(0)_{(1)}=1 / \mu$, where $r(t)_{(1)}$ is the failure rate of the survival function $\bar{F}_{(1)}(x)$. The result follows in light of (2.4). 
REMARK 2.2. The above result admits the following generalization:

$$
\lambda_{\left(\sum_{i=1}^{k+1} r_{i}+k+2\right)} \leq \lambda_{\left(r_{1}+2\right)}[\mu]^{\sum_{i=2}^{k+1} r_{i}+k} .
$$

We state the following interesting special cases:

Corollary 2.4. If $r=s=0$, then $\mu_{(3)} \leq 3 \mu_{(2)} \mu$.

Corollary 2.5. If $s=0$, then $\mu_{(r+3)} \leq(r+3) \mu_{(r+2)} \mu$.

Corollary 2.6. If $r=0$, then $\mu_{(s+3)} \leq \frac{(s+3) !}{2} \mu_{(2)}(\mu)^{s+1}$.

REMARK 2.3. Several authors derived moment inequalities for different families of life distributions such as IFR, IFRA, NBU, NBUE, DMRL and HNBUE (see e.g. [3], [4], and [9]). Thus the above results contribute to the literature for the NBUE and NBUFR classes.

3. Existence of moments generating functions. In this section, we show that the moments generating function (mgf) of $X$ exists and is finite for the NBUFR class if $\mu_{(1)}$ exists. Actually, an upper bound of the mgf is given. Precisely, we have the following result.

THEOREM 3.1. If $F$ is NBUFR and if $\mu<\infty$, then for all nonnegative $\theta \neq r(0)$,

$$
\varphi(\theta) \leq 1+\frac{\mu \theta}{1-\theta / r(0)}
$$

where $\varphi(\theta)=E\left(e^{\theta X}\right)$.

Proof. Note that

$$
\varphi(\theta)=1+\theta \int_{0}^{\infty} e^{\theta x} \bar{F}(x) d x
$$

for

$$
1+\theta \int_{0}^{\infty} e^{\theta x} \bar{F}(x) d x=1+\theta E \int_{0}^{X} e^{\theta x} d x=1+E\left(e^{\theta X}-1\right)=\varphi(\theta) .
$$

Since $\bar{F}$ is NBUFR, we have

$$
\int_{0}^{\infty} \int_{0}^{\infty} e^{\theta x} \bar{F}(x+t) d x d t \leq \int_{0}^{\infty} e^{\theta x} \bar{F}(x) d x \int_{0}^{\infty} e^{-r(0) t} d t .
$$

The left-hand side of (3.1) is equal to

$$
\begin{aligned}
\int_{0}^{\infty} \int_{0}^{u} e^{\theta(u-r)} \bar{F}(u) d r d u & =\frac{1}{\theta}\left[\int_{0}^{\infty} e^{\theta u} \bar{F}(u) d u-\mu_{(1)}\right] \\
& =\frac{1}{\theta^{2}}(\varphi(\theta)-1)-\frac{\mu}{\theta} .
\end{aligned}
$$


The right-hand side of (3.1) is equal to

$$
\frac{1}{r(0)} \int_{0}^{\infty} e^{\theta x} \bar{F}(x) d x=\frac{1}{r(0)} \frac{1}{\theta}(\varphi(\theta)-1) .
$$

The result follows from (3.2) and (3.3).

It follows from the above result that if $F$ is NBUFR and if $\mu<\infty$ then all moments of $F$ exist and are finite.

4. Estimating NWUFR survival functions. In this section, we address the question of estimating $\bar{F}(x)$ given that it is NWUFR. Let $X_{1}, \ldots, X_{n}$ denote a random sample from a life distribution $F$. With no restrictions on $F$, the empirical df $F_{n}(x)=n^{-1} \sum_{i=1}^{n} I\left(x-X_{i}\right), I(x)=1$ if $x \geq 0$ and 0 otherwise, is a widely used nonparametric estimate of $F(x)$. When $F$ is NWUFR, we shall modify $F_{n}(x)$ and offer an estimate that is NWUFR. To this end we need the following simple result.

Lemma 4.1. Let $F$ be a $d f$ and define $\bar{F}^{*}(x)=\inf _{t>0} \bar{F}(x+t) e^{r(0) t}$. Then $F^{*}=1-\bar{F}^{*}$ is NWUFR.

Proof. Note that

$$
\begin{aligned}
\bar{F}^{*}(x+y) & =\inf _{t>0} \bar{F}(x+y+t) e^{r(0) t}=\inf _{t>0} \bar{F}(x+y+t) e^{r(0)(y+t)} e^{-r(0) y} \\
& =\inf _{u>y} \bar{F}(x+u) e^{r(0) u} e^{-r(0) y} \geq \inf _{u>0} \bar{F}(x+u) e^{r(0) u} e^{-r(0) y} \\
& =\bar{F}^{*}(x) e^{-r(0) y} .
\end{aligned}
$$

We then propose to estimate $\bar{F}(x)$ by

$$
\bar{F}_{n}^{*}(x)=\inf _{t>0} \bar{F}_{n}(x+t) e^{r(0) t} .
$$

Note that $\bar{F}_{n}^{*}(x)$ is NWUFR and also that for computational needs one can write

$$
\bar{F}_{n}^{*}(x)=\min _{1 \leq i \leq n} \bar{F}_{n}\left(x+X_{(i)}\right) e^{r(0) X_{(i)}},
$$

where $X_{(1)}, \ldots, X_{(n)}$ are order statistics. Note also that the estimate in (4.1) or (4.2) assumes that $r(0)$ is known. Let us address this case first; then we will show how to deal with the case when $r(0)$ is unknown. To show the consistency of $\bar{F}_{n}^{*}(x)$ and its rate of convergence, the following lemma is essential.

Lemma 4.2. Let $D_{n}=\sup _{x}\left|F_{n}(x)-F(x)\right|$. Then for any $x \geq 0$,

$$
\left|\bar{F}_{n}^{*}(x)-\bar{F}(x)\right| \leq 3 D_{n} .
$$


Proof. Recall that $e^{r(0) t} \geq 1$ for all $t \geq 0$ and $\bar{F}_{n}(x+t) \geq \bar{F}_{n}(x) e^{-r(0) t}$ for NWUFR. Hence

$$
\begin{aligned}
\mid \bar{F}_{n}^{*}(x)- & \bar{F}(x)\left|=\inf _{t>0}\right| \bar{F}_{n}(x+t) e^{r(0) t}-\bar{F}(x) \mid \\
= & \inf _{t>0}\left|\left[\bar{F}_{n}(x+t)-\bar{F}(x) e^{-r(0) t}\right] e^{r(0) t}\right| \\
= & \inf _{t>0}\left|\left[\bar{F}_{n}(x+t)-\bar{F}(x) e^{-r(0) t}+\bar{F}_{n}(x) e^{-r(0) t}-\bar{F}_{n}(x) e^{-r(0) t}\right] e^{r(0) t}\right| \\
= & \inf _{t>0} \mid\left[\bar{F}_{n}(x+t)-\bar{F}(x+t)+\bar{F}(x+t)-\bar{F}(x) e^{-r(0) t}\right. \\
& \left.+\bar{F}_{n}(x) e^{-r(0) t}-\bar{F}_{n}(x) e^{-r(0) t}\right] e^{r(0) t} \mid \\
= & \left.\quad+\left[\bar{F}(x+t)-\bar{F}_{n}(x) e^{-r(0) t}\right]\right\} e^{r(0) t} \mid \\
= & \quad+\left|\left[\bar{F}(x+t)-\bar{F}_{n}(x) e^{-r(0) t}\right] e^{r(0) t}\right| \\
\leq \mid\left[\bar{F}_{n}(x+t)-\bar{F}(x+t)\right]+ & {\left[\bar{F}_{n}(x) e^{-r(0) t}-\bar{F}(x) e^{-r(0) t}\right] } \\
\leq &
\end{aligned}
$$

THEOREM 4.1.

(i) Let $r \geq 1$ be an integer. Then

$$
E\left|\bar{F}_{n}^{*}(x)-\bar{F}(x)\right|^{r} \leq r(9 / 2)^{r / 2} \Gamma(r / 2) n^{-r / 2} .
$$

(ii) $\left|\bar{F}_{n}^{*}(x)-\bar{F}(x)\right|=O_{w p 1}\left(n^{-1 / 2}(\ln \ln n)^{1 / 2}\right)$.

Proof. (i) Note that from Lemma 4.2, $E\left|\bar{F}_{n}^{*}(x)-\bar{F}(x)\right|^{r} \leq 3^{r} E\left(D_{n}^{r}\right)$. But using Corollary 3 of [8], we have

$$
\begin{aligned}
E\left(D_{n}^{r}\right) & =r \int_{0}^{\infty} x^{r-1} P\left(D_{n}>x\right) d x=r \int_{0}^{\infty} x^{r-1} P\left(n^{1 / 2} D_{n}>n^{1 / 2} x\right) d x \\
& =r n^{-r / 2} \int_{0}^{\infty} u^{r-1} P\left(n^{1 / 2} D_{n}>u\right) d u \\
& \leq 2 r n^{-r / 2} \int_{0}^{\infty} u^{r-1} e^{-2 u^{2}} d u=\frac{r n^{-r / 2}}{2^{r / 2}} \Gamma(r / 2) .
\end{aligned}
$$

The result is now immediate.

(ii) follows directly from Lemma 4.2 and the standard law of iterated logarithm for $D_{n}$ (see [7, p. 207]).

In the following result, we give the rate of convergence of the moment generating function of $\left|\bar{F}_{n}^{*}(x)-\bar{F}(x)\right|$. 
TheOrem 4.2. For any $\theta>0$,

$$
E e^{\theta \sqrt{n}\left|\bar{F}_{n}^{*}(x)-\bar{F}(x)\right|} \leq 1+3 \sqrt{2 \pi} \theta e^{18 \theta^{2}} .
$$

Proof. Using Lemma 4.2, we get

$$
E e^{\theta \sqrt{n}\left|\bar{F}_{n}^{*}(x)-\bar{F}(x)\right|} \leq E e^{3 \theta \sqrt{n} D_{n}} .
$$

Note that by Corollary 3 of [8],

$$
E e^{3 \theta \sqrt{n} D_{n}}=1+3 \theta \int_{0}^{\infty} e^{\theta x} P\left(n^{1 / 2} D_{n}>x\right) d x \leq 1+6 \theta \int_{0}^{\infty} e^{\theta x} e^{-2 x^{2}} d x .
$$

The result follows via integration.

Before closing this section, let us briefly discuss the case when $r(0)$ is unknown. Often $r(0)$ is a function of a set of parameters and hence could be estimated.

For example, if $\bar{F}(x ; \mu, \theta)=\exp \left\{-\left(x / \mu+\theta\left(x+e^{-x}-1\right)\right)\right\}$, known as the Makeham distribution, with the hazard rate $h(x)=1 / \mu+\theta\left(1-e^{-x}\right)$, then the value of $r(0)=1 / \mu$ could be estimated from the data.

5. Hypotheses testing. In this section, we want to test $H_{0}: F$ is exponential against $H_{1}: F$ is NBUFR and not exponential. Again we assume that $r(0)$ is known and hence take it to be one. Thus under $H_{1}, F$ satisfies

$$
\bar{F}(x+y) \leq \bar{F}(x) e^{-y}, \quad x, y \geq 0 .
$$

In the spirit of the pioneering work of [6] on the NBU class, one can take $\Delta$ as a measure of departure from $H_{0}$, where

$$
\Delta=\int_{0}^{\infty} \int_{0}^{\infty}\left[\bar{F}(x) e^{-y}-\bar{F}(x+y)\right] d F(x) d F(y) .
$$

The test based on $\Delta$ is scale invariant, so we take $\mu=1$. If $X_{1}, \ldots, X_{n}$ is a random sample from $F$, then $\Delta$ may be estimated by plugging the empirical df $F_{n}$ in place of $F$. An equivalent but much simpler approach is to weigh in (5.1) by the null df which is the exponential with mean one.

Thus we take the measure of departure from $H_{0}$ to be

$$
\delta_{1}^{*}=\int_{0}^{\infty} \int_{0}^{\infty}\left[\bar{F}(x) e^{-y}-\bar{F}(x+y)\right] e^{-x-y} d x d y .
$$

LEMMA 5.1. We can write

$$
\delta_{1}^{*}=\frac{1}{2}\left(E e^{-X}+2 E X e^{-X}-1\right) .
$$

Proof. Note that

$$
\delta_{1}^{*}=\frac{1}{2} \int_{0}^{\infty} \bar{F}(x) e^{-x} d x-\int_{0}^{\infty} u \bar{F}(u) e^{-u} d u
$$


But

$$
\int_{0}^{\infty} \bar{F}(x) e^{-x} d x=E \int_{0}^{X} e^{-x} d x=1-E e^{-X}
$$

Next,

$$
\int_{0}^{\infty} u \bar{F}(u) e^{-u} d u=E \int_{0}^{X} u e^{-u} d u=1-E e^{-X}-E X e^{-X} .
$$

The result follows from (5.2) and (5.3).

We thus take $\delta_{1}=E e^{-X}+2 E X e^{-X}-1$ as a measure of departure from $H_{0}$. This measure is estimated by

$$
\hat{\delta}_{1}=\frac{1}{n} \sum_{i=1}^{n}\left(e^{-X_{i}}+2 X_{i} e^{-X_{i}}-1\right) .
$$

Note that $\hat{\delta}_{1}$ is 0 under $H_{0}$ and is positive under $H_{1}$. We also see that $\sqrt{n}\left(\hat{\delta}_{1}-\delta_{1}\right)$ is asymptotically normal, with mean 0 and variance $\tau^{2}=$ $\operatorname{Var}\left(2 X e^{-X}+e^{-X}-1\right)$. Under $H_{0}, \delta_{1}=0$ and $\tau_{0}^{2}=2 / 27$. To do the test, reject $H_{0}$ if $\sqrt{n} \hat{\delta}_{1} /(2 / 27)^{1 / 2} \gg Z_{\alpha}$, the normal variate.

To assess the goodness of this procedure, we evaluate its Pitman Asymptotic Efficacy (PAE) and compare it to the values of the PAE of other tests of this problem. Since there are no other tests for this problem, we may compare the PAEs with those of smaller classes like NBU. The PAE is defined in this case as

$$
\operatorname{PAE}\left(\Delta_{1 \theta_{0}}\right)=\left|\int_{0}^{\infty} e^{-x} f_{\theta_{0}}^{\prime}(x) d x+2 \int_{0}^{\infty} x e^{-x} f_{\theta_{0}}^{\prime}(x) d x\right| / \tau_{0},
$$

where $f_{\theta_{0}}^{\prime}(x)=\left.\frac{d f_{\theta}(x)}{d \theta}\right|_{\theta \rightarrow \theta_{0}}$. Consider the following commonly used alternative distributions:

(i) the linear failure rate: $f_{\theta}(x)=(1+\theta x) e^{-x-\theta^{2} / 2}$,

(ii) the Makeham: $f_{\theta}(x)=\left(1+\theta\left(1-e^{-x}\right)\right) e^{-x-\theta\left(e^{2}+x-1\right)}$.

Calculating the PAEs of these alternative distributions, we get the values 0.919 and 0.510 respectively. The corresponding values of the [6] test for the NBU are 0.580 and 0.25582 and those of [2] are 0.8065 and 0.28544 . Hence our test, which deals with the much larger class NBUFR, is better and also simpler.

Acknowledgements. The authors would like to express their sincere appreciation for the valuable comments of the anonymous referee which improved the quality of this work, and for the editors of Applicationes Mathematicae for their cooperation and help. 
This work was supported by the U.S.-Egypt Science and Technology Joint Fund in cooperation with NSF, and NRC under project OTH8-020-0.

\section{References}

[1] A. Abouammoh and A. N. Ahmed, The new better than used failure rate class of life distribution, Adv. Appl. Probab. 20 (1988), 237-240.

[2] I. A. Ahmad, A class of statistics useful in testing increasing failure rate average and new better than used life distributions, J. Statist. Plann. Inference 41 (1994), 141-149.

[3] -, Moments inequalities of aging families of distributions with hypothesis testing applications, ibid. 92 (2001), 121-132.

[4] I. A. Ahmad and A. R. Mugdadi, Further moment inequalities of life distributions with hypothesis testing applications: the IFRA, NBUC, DMRL classes, ibid. 120 (2004), 1-12.

[5] J. Deshpande, S. Kochar and H. Singh, Aspects of positive ageing, J. Appl. Probab. 23 (1986), 748-758.

[6] M. Hollander and F. Proschan, Testing whether new is better than used, Ann. Math. Statist. 43 (1972), 1136-1146

[7] A. F. Karr, Probability, Academic Press, New York, 1993.

[8] P. Massart, The tight constant in the Dvoretzky-Kiefer-Wolfowitz inequality, Ann. of Probab. 18 (1990), 1269-1283.

[9] G. Reijns and A. van Gemund, Reliability analysis of hierarchical systems using statistical moments, IEEE Trans. Reliability 56 (2007), 525-533.

E. M. Shokry

Ministry of Communications

and Information Technology

Cairo, Egypt

E-mail: eshokry@mcit.gov.eg

A. N. Ahmed

Department of Mathematical Statistics

The Institute of Statistical Studies and Research

Cairo University

Cairo, Egypt

E-mail: profhadi@mailer.eun.eg
E. A. Rakha and H. M. Hewedi Department of Mathematics Faculty of Science, Suez Canal University Suez, Egypt E-mail: eiscis@hotmail.com hamedhewedi@yahoo.com

Received on 2.7.2008;

revised version on 14.1.2009 
